\title{
Spinal Epidural Lipomatosis: A Comprehensive Review
}

Paul B. Walker, BS ${ }^{1}$, Cain Sark, BS², Gioe Brennan, BS², Taylor Smith, BS², William F. Sherman, MD, MBA ${ }^{3}$, Alan D. Kaye, MD, Ph.D. ${ }^{4}$

${ }^{1}$ Weill Cornell Medical College, ${ }^{2}$ School of Medicine, Louisiana State University Health Science Center Shreveport, ${ }^{3}$ Department of Orthopaedic Surgery, Tulane University School of Medicine, ${ }^{4}$ Department of Anesthesiology, Louisiana State University Health Science Center Shreveport Keywords: spinal decompression, lumbar epidural lipomatosis, obesity, steroid-induced spinal epidural lipomatosis, spinal epidural lipomatosis https://doi.org/10.52965/001c.25571

\section{Orthopedic Reviews}

Vol. 13, Issue 1, 2021

\section{Purpose of review}

This is a comprehensive review regarding the epidemiology, diagnosis, and management of spinal epidural lipomatosis (SEL).

\section{Recent Findings}

SEL is a relatively rare condition that has gained scientific relevance over the past few decades. Recent findings include expanding treatment strategies to include minimally invasive surgical techniques.

\section{Summary \\ SEL is caused by an excess of adipose tissue accumulation localized to the thoracic and lumbar regions of the spine. While the exact pathogenesis is not fully elucidated, the etiology of SEL can be broadly classified based on five commonly associated risk factors; exogenous steroid use, obesity, endogenous steroid hormonal disease, spine surgery, and idiopathic disease. Progression of SEL may lead to neurological deficits, myelopathy, radiculopathy, neurogenic claudication, loss of sensation, difficulty voiding, lower extremity weakness, and rarely cauda equina syndrome. Conservative management is largely patient-specific and aimed at mitigating symptoms that arise from shared risk factors. If more advanced treatment measures are necessary, minimally invasive surgery and open surgical techniques, have proven successful.}

\section{INTRODUCTION}

Spinal epidural lipomatosis (SEL) is a rare condition characterized by an overgrowth of unencapsulated adipose tissue in the extradural space. ${ }^{1,2}$ This pathologic process leads to narrowing of the spinal canal and compression of surrounding neural structures. ${ }^{2}$ Clinical presentation includes myelopathy, radiculopathy, neurogenic claudication, loss of sensation, difficulty voiding, lower extremity weakness, and rarely cauda equina syndrome. ${ }^{3}$ The most common cause of SEL is long-term exogenous steroid therapy. SEL is also associated with obesity, overproduction of endogenous steroids, surgery, or deemed idiopathic. ${ }^{1,4}$ Timely management is imperative to avoid extensive disease progression and to preserve one's quality of life. Management of SEL is patient-specific and aimed at treating the underlying cause of disease. Conservative treatment includes reduction or discontinuation of offending steroid medications and weight loss. In those with persistent or severe symptoms, surgical decompression is indicated. The purpose of this review is to provide a comprehensive update on the epidemiology, pathogenesis, and management of SEL.

\section{METHODS}

A review of the current literature was performed in PubMed, Google Scholar, Embase, and Medline from January 1, 1975, to April 31, 2021. Search terms included epidural lipomatosis, spinal epidural lipomatosis, lumbar epidural lipomatosis, spinal decompression, steroids, obesity, and minimally invasive. We sought to identify reviews, prospective, retrospective, and original studies. Analysis was performed to

\footnotetext{
a Corresponding author:

Paul Walker

Weill Cornell Medical College

New York, NY

Phone: (951) 375-2109

Pbw4001@med.cornell.edu
} 
further clarify the epidemiology, pathophysiology, diagnostic workup, and treatment of SEL.

\section{CLINICAL PRESENTATION}

Symptoms of SEL are non-specific and most commonly present as myelopathy, radiculopathy, sensory disturbances, or claudication. 5,6 While some patients may have back pain, weakness, paresthesias, or ataxia early on, others may remain asymptomatic until their disease has progressed to late stages. ${ }^{7,8}$ On rare occasions, cauda equina syndrome and acute paraplegia have been the presenting signs. $3,9,10$ The wide range of symptoms is partly due to adipose accumulation in different regions of the spinal canal. ${ }^{1}$ Symptoms may develop acutely; although, most cases develop over months to years. ${ }^{11-13}$ Praver et al. found that higher severity of presenting symptoms was associated with a greater likelihood of delayed recovery. ${ }^{14}$

\section{EPIDEMIOLOGY}

The first case of SEL was reported by Lee et al. in 1975 in a patient on long-term exogenous corticosteroids following renal transplant. ${ }^{15}$ In 2015 , Theyskens et al. assessed 28,902 MRI scans and found the overall prevalence of SEL to be $2.5 \%$. Stratified further, they reported prevalence of SEL with spine-related symptoms, $1.8 \%$, incidental SEL, $0.6 \%$, and SEL with SEL-specific symptoms, $0.1 \% .{ }^{6}$ Another study assessed 831 patients with spinal stenosis. They estimated the overall prevalence of SEL to be $6.26 \%$ and the annual incidence to be $2.5 \% .{ }^{16}$ Most recently, a study performed in Korea found the prevalence of SEL to be $1.1 \%$, with a higher prevalence in males and overweight patients. ${ }^{5}$ Before these studies, Borré et al. reviewed 2,528 MRI scans and estimated a prevalence of $21 \%{ }^{17}$ The distinction between Borré et al. and the three more recent studies was the criterion for diagnosis. Borré et al. diagnosed SEL based on a rudimentary accumulation of epidural fat in the spinal canal, which they termed grade 1 . The two more recent studies only included patients with a distinct excess of epidural adipose tissue, excluding patients considered to be Borré Grade 1. Thus, the more stringent diagnostic criteria are likely more accurate and better approximates the true prevalence of SEL in the population. However, further research is needed to truly appraise the prevalence of SEL.

SEL typically occurs more commonly in males than females. $5,6,16,18-20$ Although rare, the condition has also been reported in children. One study assessed 125 children with renal disease, five out of 125 patients were diagnosed with SEL, and all five children were on methylprednisolone pulse therapy. ${ }^{21}$ The youngest in the cohort was five years old. Other studies have also reported on children with SEL. ${ }^{22-24}$ A unique presentation was in an 18-month child with no steroid use or endocrinopathies. The patient had an MRI performed for a delay in walking and was found to have SEL. This discovery may have been incidental; he was treated with conservative management and was still unable to ambulate at the time of publication.

\section{PATHOGENESIS}

The underlying pathogenesis of SEL remains largely unknown; however, irrespective of the mechanism, the condition arises due to an excessive accumulation of adipose tissue in the spinal canal. ${ }^{25-27}$ Epidural fat accumulation typically occurs slowly and will demonstrate a range of symptoms over time. ${ }^{28}$ In the initial stages, the thecal sac is diminished until it is gradually obliterated in late stages. ${ }^{28}$ Adipose deposition may cause mass effect or venous engorgement, leading to spinal cord or nerve root compression. ${ }^{29,30}$ Of note, a small mass can easily compress the thoracic cord due to its narrow width, limited vascularity, and a more significant proportion of epidural fat. ${ }^{18}$

Though SEL is exclusively localized posterior to the cord in the thoracic (T4-T8) or lumbar (L4-L5) vertebrae, the adipose tissue characteristically deposits in different locations depending on the etiology of the disease. ${ }^{29,31}$ A study by Fogel et al. reports that $55.8 \%$ of SEL cases caused by exogenous steroids affect the thoracic spine, compared to $32.7 \%$ that only involves the lumbosacral region and $11.5 \%$ that affects both. This differs from the endogenous steroid disease-related SEL, which affects the thoracic and lumbosacral areas relatively the same. In addition, the majority of obesity-related SEL (69.6\%) and idiopathic-related SEL (50\%) results in lumbosacral involvement. ${ }^{26}$ It is still unclear why specific etiologies have preferences for different locations of the spine.

\section{RISK FACTORS}

\section{EXOGENOUS STEROIDS}

Exogenous steroid use is considered the most common cause of SEL. ${ }^{1}$ Steroids stimulate glucocorticoid receptors in adipose tissue due to overlap between receptors. ${ }^{32,33}$ As such, chronic use of steroids hypertrophies spinal adipose tissue, leading to neural impingement and compressive spinal cord pathology. ${ }^{12,31}$ SEL has been documented in conditions commonly managed with exogenous steroids such as organ transplantation, Crohn's disease, nephritic syndrome, prostatic cancer, pineoblastoma, lichen ruber planus, cerebral lymphoma, diabetes mellitus, multiple sclerosis, COPD, and ulcerative colitis. ${ }^{26,31,34-39}$ Several studies estimate that around $50 \%$ of SEL cases were associated with exogenous steroid use. ${ }^{4,26}$ More recently, it was reported that $27 \%$ of cases received exogenous steroids. ${ }^{16}$

Of note, one study found a positive correlation between the number of steroid injections and SEL incidence. ${ }^{40}$ However, in a subsequent study, a mean cumulative dose of steroids needed to initiate SEL could not be determined. ${ }^{14}$ This remains a topic of investigation.

\section{OVERPRODUCTION OF ENDOGENOUS STEROIDS}

An overproduction of endogenous steroids is also associated with the development of SEL. Cushing syndrome, carcinoid tumor, hypothyroidism, and pituitary prolactinoma have all been associated with SEL. ${ }^{41-47}$ In contrast to exogenous steroids, Fogel et al. estimated that only $3.2 \%$ of cases were related to endogenous hormonal steroid dis- 
ease. ${ }^{26}$ The pathogenesis is analogous to that of exogenous steroids. Excess steroids lead to expansion of adipose tissue in the Cushingoid fat distribution and epidural space, which compresses surrounding neural structures. ${ }^{31}$

\section{OBESITY}

Obesity is considered the most common cause of SEL unrelated to steroid use. ${ }^{1,26,48}$ It was found that $24.5 \%$ of SEL cases were associated with obesity-related factors. ${ }^{26}$ Obesity is thought to cause chronic inflammation and subsequent hypertrophy of adipose tissue in the spinal canal. 1,49,50 The levels of inflammatory cytokines such as TNF-alpha and IL-beta were significantly elevated (roughly 2.6-fold) in obese patients with SEL. ${ }^{50}$ These cytokines likely enhance adipocyte growth, which explains the increase in adipocyte size in the epidural region compared to the non-obese control group. Borré et al. reported $80 \%$ of patients as obese, Sugaya et al. reported $60 \%$ of cases as obese, and Malone et al. reported $79 \%$ of cases as obese. ${ }^{16,17,51}$

Although obesity is a widely accepted risk factor for SEL, some studies have found no correlation between BMI and SEL. For example, in 2016, 28 patients were analyzed, 14 controls with degenerative disk disease, and 14 patients with SEL. 52 The results showed no statistical significance between SEL and BMI, medical comorbidities, steroid injection, or endogenous steroid disease. In another study, there was no statistical significance between the thickness of adipose tissue in the spinal canal and BMI or waist circumference. 53

\section{SPINE SURGERY}

Spine surgery has been shown to increase adipose tissue accumulation in the epidural space. Greenish et al. reported a case of SEL acutely after spinal canal decompression surgery at L4/L5. ${ }^{11}$ The patient initially presented with claudication and bilateral leg pain, which resolved immediately after surgery and led to early discharge. ${ }^{11}$ Two days later, the patient re-presented with difficulty walking and bilateral leg pain. A clinical diagnosis of an epidural hematoma was considered; however, postoperative MRI showed an accumulation of epidural fat at L5/S1, which was not present pre-operatively. ${ }^{11}$ The patient underwent a decompression of L5/S1 for SEL, with no signs of disease at three weeks and a five-month follow-up. Another account of rapidly progressive SEL was reported by Youn et al. in a 67-year-old man. He underwent endoscopic posterior foraminotomy at L4-S1. One month later, the patient re-developed leg pain. MRI showed epidural fat posterior to L5. Lipomatosis was removed via endoscopic posterior decompression at L3-L5 and his symptoms resolved. ${ }^{13}$ These cases are unique as SEL developed acutely after spine surgery.

Similarly, Choi et al. reported a case of SEL after surgery for symptomatic spondylolisthesis. ${ }^{12}$ The patient had a laminectomy with herniated disk removal at L3/L4 and anterior lumbar fusion at L5/S1. He had a preoperative BMI of 25.5 and only two treatments with epidural steroids before the operation. During the procedure, there were no signs of excess epidural fat. The patient had complete pain res- olution after surgery; however, five months later, he developed symptoms of back pain, radiating leg pain, and claudication. MRI showed extensive epidural lipomatosis of the lumbosacral spine, constrictive compression of the thecal sac, and a complete block below L4/L5. After debulking the epidural fat, his symptoms improved. Choi et al. also documented a case of SEL following percutaneous vertebroplasty at T11 and L2. ${ }^{12}$ The patient's BMI were only 22.5, with normal basal hormone levels. He had received one dose of epidural steroids one month postoperatively. The patient re-presented five months later with symptomatic SEL. MRI demonstrated compression fractures of L3 and L5 and extensive lipomatosis encasing the thecal sac in the lumbosacral spine. MR myelography showed a narrowing of L5/ S1. ${ }^{12}$

\section{IDIOPATHIC}

SEL may develop as a result of idiopathic disease. However, the definition of idiopathic is controversial. Some authors define idiopathic to suggest unknown origins, while others have used it to indicate disease caused by obesity. However, most authors refer to idiopathic as SEL in non-obese patients with unknown cause of origin. Using this definition, literature has shown that $17 \%$ of SEL cases are idiopathic, indicating a need for further research to identify more plausible inducers of SEL. ${ }^{26}$ One study reported aberrant lipid metabolism to be related to idiopathic SEL. ${ }^{54}$ However, these patients were also obese, again highlighting the controversial definition of idiopathic disease. ${ }^{26}$

While not an established risk factor, concomitant spinal neurosarcoidosis with SEL has been reported. ${ }^{55}$ Authors postulate a possible link in pathogenesis due to inflammatory factors such as TNF- $\alpha$ and IL- $1 \beta$ in both diseases.

\section{DIAGNOSIS}

The diagnosis of SEL can be challenging given that symptoms often resemble other common etiologies such as vertebral and disc disease. Given this overlap, diagnosis requires a high degree of clinical suspicion and various diagnostic modalities. The most sensitive test is advanced imaging, specifically $\mathrm{T} 1$-weighted magnetic resonance imaging (MRI). ${ }^{56}$ Historically, the "Y" sign or polygonal deformations of the dural sac have been specific for SEL. These pathognomonic signs on MRI are caused by thecal sac compression due to excess epidural fat. ${ }^{17,57,58}$ However, recent studies have indicated that the "Y" sign does not become apparent until the disease has progressed to severe stages. One specific study found a lower rate of "Y" signs in symptomatic patients compared to the initial research conducted by Kuhn et al., bringing into question the sensitivity of this feature on imaging. ${ }^{58}$ In a study conducted by Borré et al., grading patterns of SEL were defined based on the epidural fat $(\mathrm{EF})$ to spinal column (Spi C) index, with normal being $\leqslant 40 \%$ and grading of SEL being described as Grade I, II, or III. Based on this study, Grade III, characterized by an EF/ Spi C index of $\geqslant 75 \%$, is when the "Y" and other pathognomonic dural sac deformations are first seen on MRI. ${ }^{17}$ 


\section{TREATMENT AND MANAGEMENT INTRODUCTION AND OVERVIEW}

Given the varied pathogenesis underlying SEL, management requires a unique approach to each patient. ${ }^{4,8}$ Treatment typically begins conservatively; however, one study found that around $90 \%$ of patients will eventually undergo surgery. ${ }^{1}$ For those who do not respond appropriately to conservative management or suffer from severe symptoms, decompression and/or laminectomy are indicated. Outcomes of surgery depend on the spine level at which SEL is present and whether the etiology is idiopathic or secondary. Specifically, patients with idiopathic SEL at the thoracic level were noted to have the best recovery compared to secondary etiologies such as endogenous steroid use or obesity. $1,59,60$

\section{CONSERVATIVE AND MEDICAL MANAGEMENT}

Weight reduction should be pursued for obese patients, regardless of underlying etiology, as studies have shown improvement of symptoms irrespective of the cause. $1,54,61$ The degree of weight reduction for symptomatic improvement varies from patient to patient, although one study recommended a 15-kilogram reduction to improve SEL-related symptoms. ${ }^{18}$ More aggressive weight reduction methods, such as bariatric surgery and calorie-restricted diets, have also been noted to provide clinical improvement due to significant loss of epidural fat deposition. ${ }^{62}$ Overall, weight reduction may reduce the likelihood of failed management and avoid the need for surgical intervention. ${ }^{63}$

For patients on chronic corticosteroids, management is directed at a tapered corticosteroid reduction while ensuring adequate treatment of the underlying condition. However, doses as low as $15 \mathrm{mg}$ per day for as little as four months have resulted in patients developing SEL; thus, simply reducing steroid doses will not prevent SEL. ${ }^{64}$ For certain patients, increasing disease-modifying medications or utilizing alternative therapies in addition to a steroid reduction has led to symptomatic improvement. ${ }^{24}$

Management of SEL caused by endogenous steroid production depends on the exact etiology and requires a careful workup. ${ }^{1,29}$ Determining whether the condition is ACTHdependent or independent is a critical first step for patients with Cushing's disease. ACTH-dependent patients may benefit from pituitary surgery. If an ectopic ACTH-producing tumor is discovered, removal should improve symptoms. Other alternatives include medical therapy, specifically inhibitors of steroidogenesis such as metyrapone or ketoconazole. ${ }^{41}$ For patients with hypothyroidism, management is via supplementation with exogenous thyroid hormone. The overarching aim is to treat the underlying condition.

The use of epidural steroid injection as treatment remains a controversial discussion. Most studies oppose the use of steroid injection since steroids are involved in the pathogenesis of the disease, while others report successful pain management. ${ }^{16}$

\section{THERAPEUTIC ENDOSCOPIC AND MINIMALLY INVASIVE} INTERVENTION

Minimally invasive procedures are an effective option in treating SEL when conservative measures fail. ${ }^{65-68}$ Endoscopic removal of fat can be performed similarly to common endoscopic spinal surgeries such as removing a herniated nucleus pulposus. ${ }^{66}$ Minimally invasive spinal surgeries are preferred due to lower postoperative pain, shorter rehabilitation time, and less cosmetic damage when compared to more invasive surgeries. ${ }^{69}$

The first reported endoscopic procedure for SEL was performed in 1998 in a 53-year-old male patient with bilateral lower extremity pain and weakness and lower back pain. 68 The patient had undergone an L3 decompressive laminectomy ten years prior, but the procedure had not successfully relieved his pain. Evaluation of the lumbar spine with MRI revealed epidural lipomatosis from spinal levels L4 to S1. Decompression of the accumulated epidural fat was accomplished using a malleable endoscopic suction device. The author reported a much more minor surgical defect due to the endoscopic procedure. In addition, no postoperative complications were encountered, and the patient reported no back or lower extremity pain at the 6-month follow-up. ${ }^{68}$

In 2008 Sairyo et al. reported on a 71-year-old male with no prior steroid use who complained of low back and bilateral lower extremity pain, especially while standing upright. Physical and neurological examinations suggested radiculopathy of bilateral L4 nerve roots. MRI scans showed epidural fat compressing the dural sac posteriorly at L3-L4. ${ }^{66}$ Right-sided L3-L4 laminotomy. Subsequently, subsequent decompression of fat was performed endoscopically, completely removing the fatty tissue hypertrophied ligamentum flavum at L3-L4. Following surgery, the patient could walk upright and reported no lower extremity or lower back pain. No postoperative complications were reported. The surgical scar left by the endoscopic procedure measured $2.0 \mathrm{~cm}$ (Figure 1). ${ }^{66}$

Kang et al. reported three successful cases using a percutaneous bi-portal endoscopic technique (PBES) as a novel treatment of SEL. ${ }^{65}$ MRI scans before surgery revealed dural sac compression and ligamentum flavum hypertrophy in all three cases. PBES required two $0.7 \mathrm{~cm}$ skin incisions superficial to the interlaminar space. An endoscope was placed in the top portal while the necessary tools for laminotomy were guided through the lower portal (Figure 2). ${ }^{65}$ The procedure involved endoscopically guided laminotomy and ligamentum flavectomy with subsequent decompression of fat with pituitary forceps. The procedure can be performed on multiple levels of the spine during a single procedure. This method was used on a 62-year-old female with SEL compression at L4-L5. A two-level PBES was performed at levels L4-L5 and L5-S1. No postoperative complications were reported, and the patient showed improved quality of life three days after surgery. The authors also reported a successful four-level PBES on a 78-year-old male. This patient had SEL at L2-L5 on MRI scans. PBES at L2-L3, L3-L4, L4-L5, and L5-S1 was performed. Three days post-operation, MRI scans showed spinal decompression at all four levels. A third successful use of PBES was performed on a 46-year-old female diagnosed with L5-S1 epidural lipo- 
matosis. The patient underwent a two-level PBES procedure at L4-L5 and L5-S1 with no complications and improved quality of life postoperatively. 65

In 2020 Roberti et al. presented a novel case where minimally invasive tubular laminectomy was performed to drain and remove a cystic epidural lipomatosis. ${ }^{67}$ The male patient presented with bilateral lower extremity weakness and bilateral sciatica. MRI scans indicated dural sac compression by what appeared to be a cystic mass at L4-L5 (Figure 3). ${ }^{67}$ The patient elected to be treated with a minimally invasive tubular laminectomy with drainage and removal of the cystic mass. The authors reported a fatty cystic lesion with no clear capsule that was filled with yellow fluid. Pathological investigation of the cystic mass found the specimen to contain fibroadipose tissue with fat necrosis, cystic degeneration, and histiocytic inflammation. No other cases of cystic epidural lipomatosis such as this have been reported in the literature. The patient reported no complications six months post-operation. ${ }^{67}$

\section{SURGICAL MANAGEMENT}

Large-scale, controlled studies comparing the efficacies of conservative treatment, minimally invasive surgical treatment, and open surgical treatments of spinal epidural lipomatosis have not been conducted due to the condition's rarity. However, many cases showing the effectiveness of surgical management of SEL have been reported, making surgical intervention a relevant option in the treatment. ${ }^{27,30,56,60,62,70-74}$ Although, efficacy varies based on underlying etiology. Fogel et al. reported a success rate of $77 \%$ with surgery for patients who developed SEL due to exogenous steroids, while Borré et al. reported a $75 \%$ success rate. ${ }^{17,26}$ In the obese population, surgery provided relief to $67 \%$; however, weight loss and conservative management led to a greater success rate of $82 \%{ }^{26}$ For those with idiopathic disease, surgery led to symptomatic improvement in 21 out of 22 patients. ${ }^{56}$

In 2016, Ferlic et al. published an analysis of 22 patientreported outcomes of surgical spinal decompression as a treatment for MRI confirmed lumbar SEL. ${ }^{56}$ The authors used the Core Outcome Measures Index (COMI) to assess pre-and postoperative pain, function, well-being, quality of life (QOL), work disability, and social disability reported by the patients. After the 3-month postoperative COMI questionnaires, the authors reported a significant improvement $(p<.05)$ in mean $(n=22)$ scores for overall COMI, leg pain, and back pain. While $81 \%$ of the patients saw improvement in their COMI scores, only $50 \%$ of patients had improved their score more than the minimum clinically significant change (MCIC) of 2.2 points. The significantly enhanced mean COMI score remained at one year and two years $(n=20)$ post-operation compared to the preoperative COMI values. Since half the patients met the MCIC improvement in COMI scores and $81 \%$ of patients showing improvement in their COMI score, the authors report decompression surgery to be a clinically relevant option for the treatment of lumbar SEL. 56

Yasuda et al. analyzed 16 patients that underwent decompression surgery as a treatment for SEL, with the efficacy of the treatment measured using the Japanese Or-

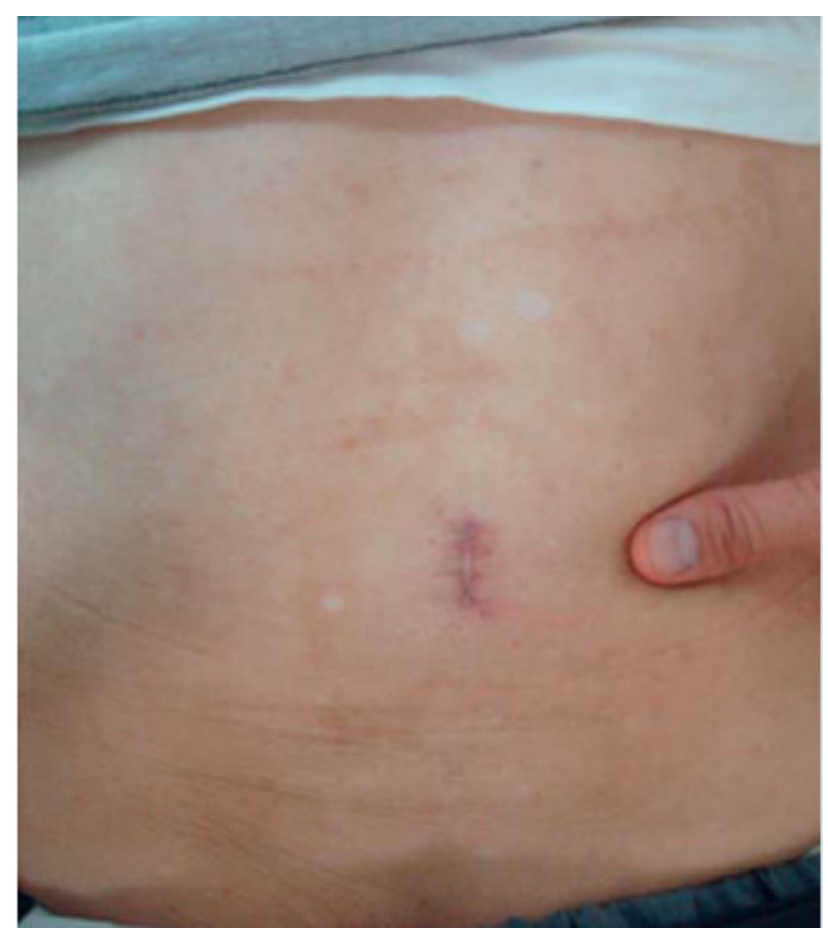

Figure 1. Surgical scar left by minimally invasive surgery. Taken from Sairyo et al. ${ }^{66}$

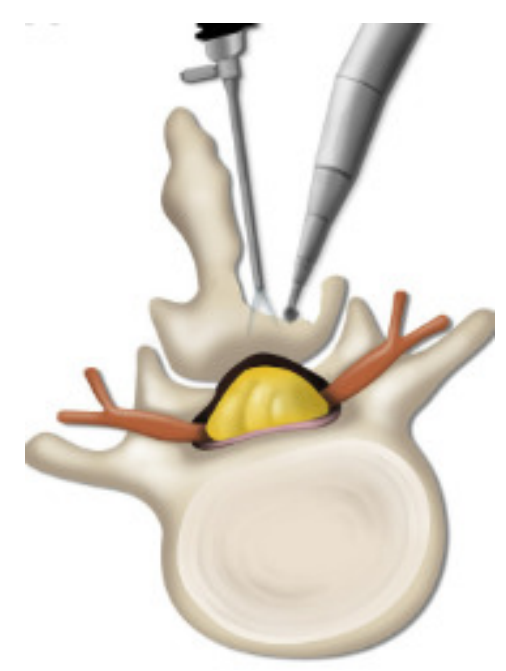

Figure 2. Illustration of PBES laminotomy technique. Taken from Kang et al. ${ }^{65}$

thopaedic Association (JOA) scores at 3, 6, and 12 months post-operation. ${ }^{27}$ The authors reported no recurrent or worsening cases upon follow-up, with a mean $(n=16)$ JOA scores improving from the preoperative value of $15.2 \pm 2.8$ to $21.3 \pm 2.2$ after three months, $24.6 \pm 1.2$ after six months, and $25.4 \pm 2.5$ twelve months after surgery.

In a review of SEL among the Korean population, Yoo et al. reported 23 cases of SEL where laminectomy with decompression was performed. The authors reported outcomes of "excellent to good" in 22 of the 23 cases, with one case resulting in mortality related to a different medical complication. ${ }^{72}$ Ferlic et al., Yasuda et al., and Yoo et al. 
conclude in their studies that surgical decompression is an effective treatment option for spinal SEL only after conservative treatments such as weight loss are proven to be ineffective. ${ }^{27,56,72}$

Weight loss induced by bariatric surgery has been shown in at least one case to resolve SEL. ${ }^{62}$ Valcarenghi et al. reported a 48-year-old male with a Body Mass Index (BMI) of 37.4 with MRI confirmed SEL at level L5-S1. The patient was not an ideal candidate for decompression surgery due to his age and weight, so a more conservative approach of a sleeve gastroplasty was performed. Seven months after the procedure, MRI scans revealed almost complete resolution of the SEL. ${ }^{62}$ Since regaining the weight is often seen long-term in bariatric surgery patients, further studies on the effects of weight regain on SEL treated with weight loss need to be conducted to make bariatric surgery a more clinically relevant treatment option for SEL. ${ }^{75}$

Since 2018 three publications have compared the outcomes of spinal decompression surgeries between patients with SEL and patients with non-SEL-induced spinal stenosis. ${ }^{60,73,74}$ Bayerl et al. conducted a three-year observational study comparing the decompression surgical outcomes of patients with SEL $(n=38)$ and patients with classic spinal stenosis (CSS) $(n=51) .{ }^{73}$ On follow-up, patients in both groups were evaluated using the Roland-Morris Disability Questionnaire, Oswestry Disability Index, Numeric Pain Scale, walking distance, and the Short Form-36 form. The authors report a comparable clinical outcome between the SEL and CSS groups at three years post-operation. During the three-year follow-up, no significant differences in any outcome scores were found between the two groups, along with $71 \%$ and $69 \%$ of patients in the SEL and CSS groups, respectively, reporting satisfaction with the surgery. ${ }^{73}$

Similar studies have reported findings inconsistent with Bayerl et al. ${ }^{6,74}$ Fujita et al. analyzed the spinal decompression surgery outcomes of patients with SEL and compared them with patients without SEL. ${ }^{60}$ All patients were assessed using the Japanese Orthopedic Association Back Pain Evaluation Questionnaire (JOABPEQ) and the RolandMorris Disability Questionnaire (RDQ). Using a method described in the publication, the authors further classified the surgical outcome as either "effective" or "not effective" during the follow-up visits. They found all but the psychological disorder domain JOABPEQ scores significantly improved in both groups at the 1- and 2-year post-operation visits compared to the preoperative values. Both groups also saw significant improvement in their RDQ scores following their operation. However, the authors' analysis found that SEL was significantly associated with a "not effective" outcome in 1-year post-op walking ability and social life domains, as well as 2-year post-op walking ability.

Ulrich et al. compared spinal decompression surgical outcomes of patients with SEL $(n=14)$ to spinal stenosis patients without SEL at 1- and 2-year follow-up. ${ }^{74}$ Pain, disability, and quality of life were assessed using Spinal Stenosis Measure (SSM) symptoms, SSM function, and EQ-5D-3L summary index (SI). The authors found that while disability was significantly improved in the patients with SEL at the 2-year post-op visit, pain and quality of life were not significantly improved. Ulrich et al. concluded that the SEL

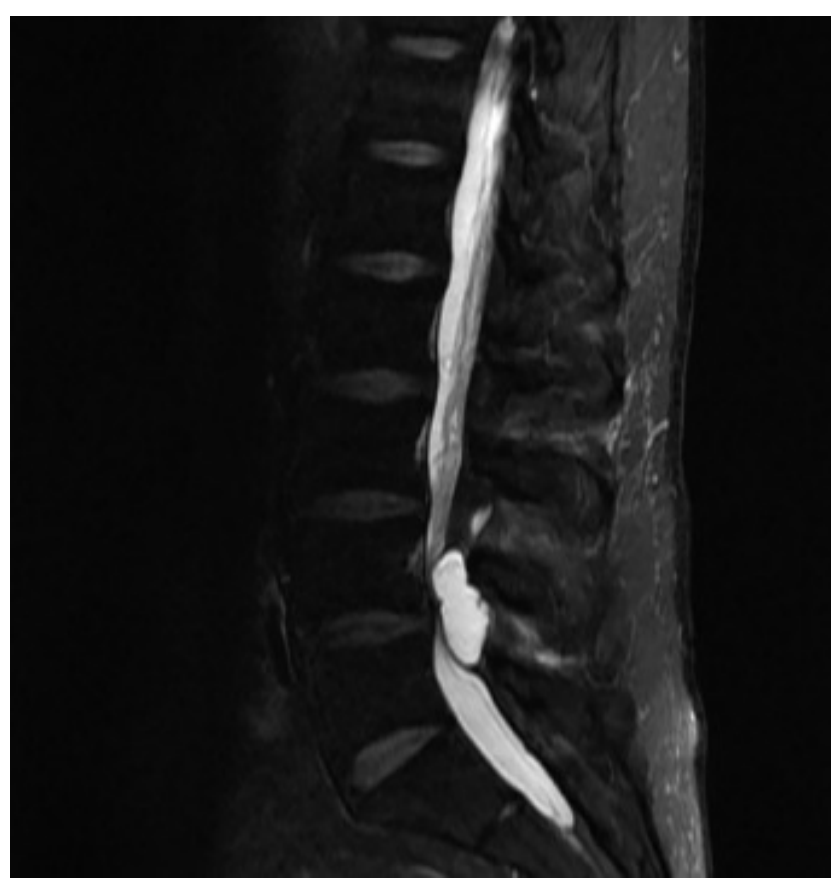

Figure 3. The cystic mass was shown on a sagittal "fat suppression" MRI scan and taken from Roberti et al. 67

group had a lower quality of life and more reported pain than those without SEL. ${ }^{74}$

Han et al. reported a novel surgical method involving internal fixation and bone graft for treatment of SEL in a 53-year-old male with a BMI of 33.3. He presented with low back pain, bilateral leg pain, and numbness. MRI showed severe lumbar spinal stenosis with hypertrophic adipose tissue and ligaments. He underwent decompression, resection of epidural adipose tissue by bipolar cautery, internal fixation, and bone graft fusion. Internal fixation and bone graft fusion help maintain the stability of vertebrae. It has been performed in obese patients with lumbar degenerative disease; however, this was the first time it has been documented as being performed in the setting of obesity-induced SEL. ${ }^{76}$ At 22 months, the patient had improved symptoms. This may be a feasible technique for SEL in appropriate cases.

\section{CONCLUSION}

Spinal Epidural Lipomatosis is a rare condition caused by excess adipose tissue in the spinal canal. The excess tissue compresses the spinal cord, leading to pain and neurologic deficits. The pathogenesis is largely unknown; however, five major risk factors have been outlined, the most common involving exogenous steroids and obesity. Diagnosis is typically made through MRI and management heavily relies on reducing any factor associated with the disease. It is most often tailored to the patient. In more severe cases, minimally invasive and open surgery has proven to be successful. While the etiology of the disease is becoming more clear, further research is needed for novel treatments and more precise practice guidelines. 


\section{AUTHOR CONTRIBUTIONS}

Sherman and Kaye conceived the topic of interest and were involved in planning and supervision. Walker performed a comprehensive review of the literature and drafted the framework. Walker, Stark, Brennan, Smith, Sherman, and Kaye contributed to the writing and editing of the manuscript.

Submitted: June 21, 2021 EST, Accepted: July 03, 2021 EST 


\section{REFERENCES}

1. Kim K, Mendelis J, Cho W. Spinal Epidural Lipomatosis: A Review of Pathogenesis, Characteristics, Clinical Presentation, and Management. Global Spine Journal. 2019;9(6):658-665. doi:10.1177/2192568218793617

2. Fassett DR, Schmidt MH. Spinal epidural lipomatosis: a review of its causes and recommendations for treatment. Neurosurgical focus. 2004;16(4):1-3. doi:10.3171/foc.2004.16.4.12

3. Okunlola AI, Orewole TO, Okunlola CK, Babalola OF, Akinmade A. Epidural lipomatosis in elderly patient: A rare cause of cauda equina compression. Surgical Neurology International. 2021;12:7. doi:10.252 59/sni_840_2020

4. Al-Khawaja D, Seex K, Eslick GD. Spinal epidural lipomatosis - A brief review. Journal of Clinical Neuroscience. 2008;15(12):1323-1326. doi:10.1016/i.jo cn.2008.03.001

5. Park SK, Han JM, Lee K, Cho WJ, Oh JH, Choi YS. The clinical characteristics of spinal epidural lipomatosis in the lumbar spine. Anesth Pain Med. 2018;8(5). doi:10.5812/aapm.83069

6. Theyskens NC, Paulino Pereira NR, Janssen SJ, Bono CM, Schwab JH, Cha TD. The prevalence of spinal epidural lipomatosis on magnetic resonance imaging. Spine Journal. 2017;17(7):969-976. doi:10.10 16/j.spinee.2017.02.010

7. Ge Y, Yang X, You Y, Xuan Y, Yan G. Comparison of relative and absolute values of magnetic resonance imaging in the diagnosis of spinal epidural lipomatosis. Journal of Spinal Cord Medicine. 2019;42(4):502-507. doi:10.1080/10790268.2018.1449 $\underline{782}$

8. Alvarez A, Induru R, Lagman R. Considering Symptomatic Spinal Epidural Lipomatosis in the Differential Diagnosis. Am J Hosp Palliat Care. 2013;30(6):617-619. doi:10.1177/1049909112457012

9. Andress HJ, Schürmann M, Heuck A, Schmand J, Lob G. A rare case of osteoporotic spine fracture associated with epidural lipomatosis causing paraplegia following long-term cortisone therapy. Archives of Orthopaedic and Trauma Surgery. 2000;120(7-8):484-486. doi:10.1007/s004029900086
10. Miwa T, Yamashita T, Sakaura H, Ohzono K, Ohwada T. Steroid-induced paraparesis: Spinal epidural lipomatosis complicated by a wedge deformity of the middle thoracic vertebrae. Intern Med. 2013;52(14):1621-1624. doi:10.2169/internalme dicine.52.9379

11. Greenish D, Watura K, Harding I. Spinal epidural lipomatosis following bilateral spinal decompression surgery. BMJ Case Rep. 2019;12(2):e226985. doi:10.11 36/bcr-2018-226985

12. Choi KC, Kang BU, Lee CD, Lee SH. Rapid progression of spinal epidural lipomatosis. Eur Spine J. 2012;21(S4):00586-00011. doi:10.1007/s00586-01 $\underline{1-1855-\mathrm{X}}$

13. Youn MS, Woo YH, Shin JK. Rapid progression of spinal epidural lipomatosis after percutaneous endoscopic spine surgery mimicking disc herniation. International Journal of Surgery Case Reports. 2020;73:1-4. doi:10.1016/j.ijscr.2020.06.083

14. Praver M, Kennedy BC, Ellis JA, D’Amico R, Mandigo CE. Severity of presentation is associated with time to recovery in spinal epidural lipomatosis. Journal of Clinical Neuroscience. 2015;22(8):1244-1249. doi:10.1016/j.jocn.2015.03.005

15. Lee M, Lekias J, Gubbay SS, Hurst PE. Spinal cord compression by extradural fat after renal transplantation. Medical Journal of Australia. 1975;1(7):201-203. doi:10.5694/j.1326-5377.1975.tb1 11328.x

16. Malone JB, Bevan PJ, Lewis TJ, Nelson AD, Blaty $\mathrm{DE}$, Kahan ME. Incidence of spinal epidural lipomatosis in patients with spinal stenosis. Journal of Orthopaedics. 2018;15(1):36-39. doi:10.1016/i.jor.201 7.11.001

17. Borré DG, Borré GE, Aude F, Palmieri GN. Lumbosacral epidural lipomatosis: MRI grading. Eur Radiol. 2003;13(7):00330-00002. doi:10.1007/s0033 0-002-1716-4

18. Robertson SC, Traynelis VC, Follett KA, Menezes AH. Idiopathic spinal epidural lipomatosis. Neurosurgery. 1997;41(1):68-75. doi:10.1097/0000612 3-199707000-00015

19. Roy-Camille R, Mazel CH, Husson JL, Saillant G. Symptomatic spinal epidural lipomatosis induced by a long-term steroid treatment: Review of the literature and report of two additional cases. Spine. 1991;16(12):1365-1371. doi:10.1097/00007632-19911 2000-00004 
20. Stern JD, Quint DJ, Sweasey TA, Hoff JT. Spinal epidural lipomatosis: Two new idiopathic cases and a review of the literature. Journal of Spinal Disorders. 1994;7(4):343-349. doi:10.1097/00002517-19940800 0-00009

21. Kano K, Kyo K, Ito S, et al. Spinal epidural lipomatosis in children with renal diseases receiving steroid therapy. Pediatr Nephrol. 2005;20(2):184-189. doi:10.1007/s00467-004-1723-6

22. Muñoz A, Barkovich JA, Mateos F, Simón R. Symptomatic epidural lipomatosis of the spinal cord in a child: MR demonstration of spinal cord injury. Pediatric Radiology. 2002;32(12):865-868. doi:10.100 7/s00247-002-0811-7

23. Perling LH, Laurent JP, Cheek WR. Epidural hibernoma as a complication of corticosteroid treatment: Case report. Journal of Neurosurgery. 1988;69(4):613-616. doi:10.3171/jns.1988.69.4.0613

24. Möller JC, Cron RQ, Young DW, et al.

Corticosteroid-induced spinal epidural lipomatosis in the pediatric age group: Report of a new case and updated analysis of the literature. Pediatr Rheumatol. 2011;9(1):1-6. doi:10.1186/1546-0096-9-5

25. Mallard F, Buni M, Nolet PS, Emary P, Taylor JA, Moammer G. Lumbar spinal epidural lipomatosis: A case report and review of the literature. International Journal of Surgery Case Reports. 2021;78:71-75. doi:1 $\underline{0.1016 / j . i j s c r .2020 .11 .128}$

26. Fogel GR, Cunningham PY III, Esses SI. Spinal epidural lipomatosis: Case reports, literature review and meta-analysis. Spine Journal. 2005;5(2):202-211. doi:10.1016/j.spinee.2004.05.252

27. Yasuda T, Suzuki K, Kawaguchi Y, et al. Clinical and imaging characteristics in patients undergoing surgery for lumbar epidural lipomatosis. $B M C$ Musculoskelet Disord. 2018;19(1). doi:10.1186/s1289 1-018-1988-8

28. Quint DJ, Boulos RS, Sanders WP, Mehta BA, Patel SC, Tiel RL. Epidural lipomatosis. Radiology. 1988;169(2):485-490. doi:10.1148/radiology.169.2.31 $\underline{74998}$

29. Papastefan ST, Bhimani AD, Denyer S, et al. Management of idiopathic spinal epidural lipomatosis: a case report and review of the literature. Childs Nerv Syst. 2018;34(4):00381-00017. $\underline{\mathrm{d}}$ oi:10.1007/s00381-017-3706-5

30. López-González A, Resurrección Giner M. Idiopathic spinal epidural lipomatosis: Urgent decompression in an atypical case. Eur Spine J. 2008;17(S2):00586-00007. doi:10.1007/s00586-007-04 $\underline{65-0}$
31. Koch CA, Doppman JL, Patronas NJ, Nieman LK, Chrousos GP. Do glucocorticoids cause spinal epidural lipomatosis? When endocrinology and spinal surgery meet. Trends in Endocrinology \& Metabolism. 2000;11(3):86-90. doi:10.1016/s1043-2760(00)0023 $\underline{6-8}$

32. Bamberger CM, Schulte HM, Chrousos GP. Molecular determinants of glucocorticoid receptor function and tissue sensitivity to glucocorticoids. Endocrine Reviews. 1996;17(3):245-261. doi:10.1210/e drv-17-3-245

33. Chrousos GP. A New "New" Syndrome in the New World: Is Multiple Postreceptor Steroid Hormone Resistance Due to a Coregulator Defect? The Journal of Clinical Endocrinology \& Metabolism. 1999;84(12):4450-4453. doi:10.1210/jcem.84.12.6073

34. Kotilainen E, Hohenthal U, Karhu J, Kotilainen P. Spinal epidural lipomatosis caused by corticosteroid treatment in ulcerative colitis. European Journal of Internal Medicine. 2006;17(2):138-140. doi:10.1016/j.e jim.2005.08.011

35. Gehring U, Mugele K, Ulrich J. Cellular receptor levels and glucocorticoid responsiveness of lymphoma cells. Molecular and Cellular Endocrinology. 1984;36(1-2):107-113. doi:10.1016/0303-7207(84)900 $\underline{89-3}$

36. Danielsen M, Stallcup MR. Down-regulation of glucocorticoid receptors in mouse lymphoma cell variants. Mol Cell Biol. 1984;4(3):449-453. doi:10.112 8/mcb.4.3.449-453.1984

37. Bourgeois S, Newby RF. Correlation between Glucocorticoid Receptor and Cytolytic Response of Murine Lymphoid Cell Lines. Cancer Research. 1979;39(11).

38. Pinsker MO, Kinzel D, Lumenta CB. Epidural thoracic lipomatosis induced by long-term steroid treatment case illustration. Acta Neurochirurgica. 1998;140(9):991-992. doi:10.1007/s007010050205

39. Zentner J, Buchbender K, Vahlensieck M. Spinal epidural lipomatosis is a complication of prolonged corticosteroid therapy. Journal of Neurosurgical Sciences. 1995;39(1):81-85.

40. Jaimes R III, Rocco AG. Multiple epidural steroid injections and body mass index linked with occurrence of epidural lipomatosis: A case series. BMC Anesthesiol. 2014;14(1):1-9. doi:10.1186/1471-2 253-14-70 
41. Koch CA, Doppman JL, Watson JC, Patronas NJ, Nieman LK. Spinal epidural lipomatosis in a patient with the ectopic corticotropin syndrome. $N$ Engl J Med. 1999;341(18):1399-1400. doi:10.1056/nejm1999 10283411814

42. Sivakumar K, Sheinart K, Lidov M, Cohen B. Symptomatic spinal epidural lipomatosis in a patient with Cushing's disease. Neurology.

1995;45(12):2281-2283. doi:10.1212/wnl.45.12.2281

43. Doppman JL. Epidural lipomatosis. Radiology. 1989;171(2):581-582. doi:10.1148/radiology.171.2.27 $\underline{04829}$

44. Noël P, Pepersack T, Vanbinst A, Allé JL. Spinal epidural lipomatosis in Cushing's syndrome secondary to an adrenal tumor. Neurology. 1992;42(6):1250-1251. doi:10.1212/wnl.42.6.1250

45. Bhatia K, Frydenberg E, Steel T, Ow-Yang M, Ho $\mathrm{K}$, Grainger E. Spinal epidural lipomatosis due to a bronchial ACTH-secreting carcinoid tumour. Journal of Clinical Neuroscience. 2010;17(11):1461-1462. doi:1 $\underline{0.1016 / j . j o c n .2010 .04 .008}$

46. Ahmad S, Best T, Lansdown A, et al. Spinal epidural lipomatosis: A rare association of Cushing's disease. Endocrinology, Diabetes \& Metabolism Case Reports. 2020;2020(1):1-6. doi:10.1530/edm-20-0111

47. Chaudhary K, Chandy D, Agrawal V, Dhawale A, Nagaonkar S. Spinal Epidural Lipomatosis with Osteoporotic Vertebral Fractures Presenting as Acute Thoracic Myelopathy in a 33-Year-Old Man with Cushing Disease. World Neurosurgery. 2020;142:136-141. doi:10.1016/j.wneu.2020.06.212

48. Morishita S, Arai Y, Yoshii T, Sakai K, Hirai T, Okawa A. Lumbar epidural lipomatosis is associated with visceral fat and metabolic disorders. Eur Spine J. 2018;27(7):1653-1661. doi:10.1007/s00586-018-558 $\underline{4-2}$

49. Haddad SF, Hitchon PW, Godersky JC. Idiopathic and glucocorticoid-induced spinal epidural lipomatosis. Journal of Neurosurgery. 1991;74(1):38-42. doi:10.3171/jns.1991.74.1.0038

50. Fujita N, Hosogane N, Hikata T, et al. Potential involvement of obesity-associated chronic inflammation in the pathogenesis of idiopathic spinal epidural lipomatosis. Spine.

2016;41(23):E1402-E1407. doi:10.1097/brs.000000000 $\underline{0001646}$

51. Sugaya H, Tanaka T, Ogawa T, Mishima H. Spinal epidural lipomatosis in lumbar magnetic resonance imaging scans. Orthopedics. 2014;37(4). doi:10.3928/0 1477447-20140401-57
52. Al-Omari AA, Phukan RD, Leonard DA, Herzog TL, Wood KB, Bono CM. Idiopathic spinal epidural lipomatosis in the lumbar spine. Orthopedics. 2016;39(3):163-168. doi:10.3928/01477447-2016031 5-04

53. Alicioglu B, Sarac A, Tokuc B. Does abdominal obesity cause an increase in the amount of epidural fat? Eur Spine J. 2008;17(10):1324-1328. doi:10.1007/s 00586-008-0724-8

54. Ishihara S, Fujita N, Azuma K, et al. Spinal epidural lipomatosis is a previously unrecognized manifestation of metabolic syndrome. Spine Journal. 2019;19(3):493-500. doi:10.1016/j.spinee.2018.07.022

55. Jaafar N, Khoueiry M, Khoury SJ, Makki A. Rare Case of Spinal Neurosarcoidosis with Concomitant Epidural Lipomatosis. Gontkovsky ST, ed. Case Reports in Neurological Medicine. 2021;2021:1-6. doi:1 $\underline{0.1155 / 2021 / 5952724}$

56. Ferlic PW, Mannion AF, Jeszenszky D, et al. Patient-reported outcome of surgical treatment for lumbar spinal epidural lipomatosis. Spine Journal. 2016;16(11):1333-1341. doi:10.1016/j.spinee.2016.0 $\underline{6.022}$

57. Ishikawa Y, Shimada Y, Miyakoshi N, et al. decompression of idiopathic lumbar epidural lipomatosis: Diagnostic magnetic resonance imaging evaluation and review of the literature. Journal of Neurosurgery: Spine. 2006;4(1):24-30. doi:10.3171/sp i.2006.4.1.24

58. Kuhn MJ, Youssef HT, Swan TL, Swenson LC. Lumbar epidural lipomatosis: the "Y" sign of thecal sac compression. Computerized Medical Imaging and Graphics. 1994;18(5):367-372. doi:10.1016/0895-611 1(94) $90007-8$

59. Kellett CG, Siva V, Norman ICF, Jung J, Grahovac G, Minhas P. Symptomatic Idiopathic Spinal Epidural Lipomatosis in 9 Patients: Clinical, Radiologic, and Pathogenetic Features. World Neurosurgery. 2019;126:e33-e40. doi:10.1016/i.wneu.2019.01.098

60. Fujita N, Ishihara S, Michikawa T, et al. Negative impact of spinal epidural lipomatosis on the surgical outcome of posterior lumbar spinous-splitting decompression surgery: a multicenter retrospective study. Spine Journal. 2019;19(12):1977-1985. doi:10.1 016/j.spinee.2019.06.022

61. Beckworth WJ, McCarty EJ, Garcia-Corrada JE, Holbrook JF. Epidural Lipomatosis and Associated Spinal Stenosis-The Impact of Weight Loss: A Case Report. American Journal of Lifestyle Medicine. 2017;11(6):511-514. doi:10.1177/1559827616686764 
62. Valcarenghi J, Bath O, Boghal H, Ruelle M, Lambert J. Benefits of bariatric surgery on spinal epidural lipomatosis: case report and literature review. Eur J Orthop Surg Traumatol. 2018;28(7):1437-1440. doi:10.1007/s00590-018-220 6-y

63. Kniprath K, Farooque M. Drastic weight reduction decrease in epidural fat and concomitant improvement of neurogenic claudicatory symptoms of spinal epidural lipomatosis. Pain Med. Published online January 20, 2017:pnw313. doi:10.1093/pm/pn $\underline{\mathrm{w} 313}$

64. Gupta R, Kumar AN, Gupta V, Madhavan SM, Sharma SK. An unusual cause of paraparesis in a patient on chronic steroid therapy. Journal of Spinal Cord Medicine. 2007;30(1):67-69. doi:10.1080/107902 $\underline{68.2007 .11753916}$

65. Kang SS, Lee SC, Kim SK. A Novel Percutaneous Biportal Endoscopic Technique for Symptomatic Spinal Epidural Lipomatosis: Technical Note and Case Presentations. World Neurosurgery. 2019;129:49-54. d oi:10.1016/j.wneu.2019.05.214

66. Sairyo K, Sakai T, Higashino K, Hirao B, Katoh S, Yasui N. Minimally invasive excision of lumbar epidural lipomatosis using a spinal endoscope. Minim Invasive Neurosurg. 2008;51(1):43-46. doi:10.1055/s-2 $\underline{007-1004569}$

67. Roberti F, Bell J, Arsenault K. Lumbar cystic epidural lipomatosis with mass effect. Report of an unusual case. Journal of Clinical Neuroscience. 2020;77:195-198. doi:10.1016/j.jocn.2020.05.038

68. Frank E. Endoscopic suction decompression of idiopathic epidural lipomatosis. Surgical Neurology. 1998;50(4):333-335. doi:10.1016/s0090-3019(98)0001 $\underline{6-0}$
69. Peng H, Tang G, Zhuang X, Lu S, Bai Y, Xu L. Minimally invasive spine surgery decreases postoperative pain and inflammation for patients with lumbar spinal stenosis. Exp Ther Med. 2019;18(4). doi:10.3892/etm.2019.7917

70. Bushkar J, MenkinSmith L, Diann K. Idiopathic Spinal Epidural Lipomatosis Causing Cauda Equina Syndrome. Clinical Practice and Cases in Emergency Medicine. 2017;1(4):305-308. doi:10.5811/cpcem.201 7.6.34778

71. Akhaddar A, Ennouali H, Gazzaz M, Naama O, Elmostarchid B, Boucetta M. Idiopathic spinal epidural lipomatosis without obesity: A case with relapsing and remitting course. Spinal Cord. 2008;46(3):243-244. doi:10.1038/sj.sc.3102099

72. Yoo JC, Choi JJ, Lee DW, Lee SP. Spinal epidural lipomatosis in Korean. J Korean Neurosurg Soc. 2014;55(6):365-369. doi:10.3340/jkns.2014.55.6.365

73. Bayerl SH, Dinkelbach M, Heiden P, Prinz V, Finger T, Vajkoczy P. Treatment results for lumbar epidural lipomatosis: Does fat matter? Eur Spine J. 2019;28(1):69-77. doi:10.1007/s00586-018-5771-1

74. Ulrich NH, Gravestock I, Winklhofer S, et al. Twoyear outcome comparison of decompression in 14 lipomatosis cases with 169 degenerative lumbar spinal stenosis cases: a Swiss prospective multicenter cohort study. Eur Spine J. 2020;29(9):00586-00020. do $\mathrm{i}: 10.1007 / \mathrm{s} 00586-020-06449-\mathrm{w}$

75. Sarela AI, Dexter SPL, O’Kane M, Menon A, McMahon MJ. Long-term follow-up after laparoscopic sleeve gastrectomy: 8-9-year results. Surgery for Obesity and Related Diseases. 2012;8(6):679-684. doi:1 0.1016/j.soard.2011.06.020

76. Han X, Xu D, Ren ZN, Chen X, Li Z, Li S. Lumbar spinal stenosis combined with obesity-induced idiopathic spinal epidural lipomatosis treated with posterior lumbar fusion: case report. BMC Surg. 2021;21(1). doi:10.1186/s12893-021-01157-8 